\title{
The importance of non-LTE models for the interpretation of observations of interstellar NO
}

\author{
F. Lique ${ }^{1,2, \star}$, F. F. S. van der Tak ${ }^{3}$, J. Kłos ${ }^{2}$, J. Bulthuis ${ }^{4}$, and M. H. Alexander ${ }^{2}$ \\ 1 LERMA and UMR 8112 of CNRS, Observatoire de Paris-Meudon, 92195 Meudon Cedex, France \\ e-mail: francois.lique@obspm. fr \\ 2 Department of Chemistry and Biochemistry, University of Maryland, College Park, MD 20742, USA \\ 3 SRON Netherlands Institute for Space Research, Landleven 12, 9747 AD Groningen, The Netherlands \\ e-mail:vdtak@sron.nl \\ ${ }^{4}$ Department of Physical Chemistry and Laser Centre, Vrije Universtiteit, de Boelelaan 1083, 1081 HV Amsterdam, The Netherlands
}

Received 23 June 2008 / Accepted 10 October 2008

\section{ABSTRACT}

\begin{abstract}
Context. The ALMA and Herschel missions promise to deliver data of high spatial and spectral resolution at far-infrared and submillimeter wavelengths. Modeling these data will require the knowledge of accurate radiative and collisional rates for species of astrophysical interest.

Aims. We calculate the rotational excitation rate coefficients of NO in collisions with He and check the validity of the LTE approach in interpreting observations of rotational lines of NO.

Methods. State-to-state rate coefficients between the 360 lowest hyperfine levels of NO were calculated using the $M_{J}$ randomizing limit method for temperatures from $10 \mathrm{~K}$ to $350 \mathrm{~K}$. We performed calculations of the excitation of NO using the new rate coefficients using a large velocity gradient $(\mathrm{LVG})$ radiative transfer code.

Results. The critical densities of the lines are found to be at $\gtrsim 10^{5} \mathrm{~cm}^{-3}$. The low dipole moment of NO ensures that the line emission is optically thin up to column densities of $\sim 10^{15} \mathrm{~cm}^{-2}$. Lines in the ground $(\Omega=1 / 2)$ state are readily detectable in typical conditions $\left(N(\mathrm{NO})=10^{13} \mathrm{~cm}^{-2} ; T=10-30 \mathrm{~K}\right)$, whereas lines in the excited $\Omega=3 / 2$ state are observed only in warm $(T>50 \mathrm{~K})$ regions with higher column densities $\left(N(\mathrm{NO})=10^{14} \mathrm{~cm}^{-2}\right)$. Line ratios of $\mathrm{NO}$ may well be used to constrain the ambient temperature and/or density.

Conclusions. The new rate coefficients will help significantly in interpreting NO emission lines observed with current and future telescopes, and enable this molecule to become a powerful astrophysical tool.
\end{abstract}

Key words. ISM: molecules - molecular data - radiative transfer

\section{Introduction}

Knowledge of chemical conditions in dense interstellar clouds is essential in understanding key processes in the Universe, such as the gravitational collapse of these clouds that leads to the formation of stars, and gas accretion onto supermassive black holes at the centers of many galaxies. Due to their low temperatures and high extinctions, dense interstellar clouds should be observed at long (infrared and radio) wavelengths, and their submillimeterwave emission line spectrum is a prime diagnostic tool. The use of molecular rotation lines to estimate the physical parameters of the clouds, such as volume density and kinetic temperature, requires understanding of the underlying chemical processes to understand which molecules are abundant under which conditions. Furthermore, the chemical composition of interstellar matter depends on parameters that are otherwise difficult to estimate, such as time and X-ray radiation field. By using chemical models, these parameters can be used to gain information about the clouds, which would be otherwise impossible to obtain (see Van der Tak 2005, for a review of this subject).

The estimation of molecular column densities and abundances from spectral line data at infrared or submillimeter

\footnotetext{
* Present address: LOMC - FRE 3102, CNRS-Université du Havre, 25 rue Philippe Lebon, BP 540, 76058 Le Havre, France.
}

wavelengths may be carried out at several levels of sophistication (Van der Tak et al. 2007). The choice of method depends on the nature of the observational data, but also on the available molecular data. In particular, while spectroscopic data are available for most molecules of astrophysical interest, collisional data are much rarer. Of the more than 140 molecules known to exist in space, rate coefficients for collisional (de)excitation exist for fewer than 30. The existence of accurate collision rates is crucial to the interpretation of molecular line observations. Without these rates, only approximate estimates of the molecular column density are possible assuming local thermodynamic equilibrium (LTE), which at the typical densities of dense interstellar clouds is generally not a good approximation. For molecules for which rates are known, non-LTE calculations may be performed that enable us to complete accurate column density estimates from single lines, but also estimates of kinetic temperature and volume density if several lines are observed. Therefore, astrophysical models improve significantly in sophistication with knowledge of molecular collision rates. An example of a key chemical species for which collisional excitation coefficients have been unavailable until now is NO.

Nitric oxide (NO) is proposed to be the main precursor species in interstellar $\mathrm{N}-\mathrm{O}$ chemistry, but its abundance remains poorly understood for at least two reasons. First, the main 
production $(\mathrm{N}+\mathrm{OH} \rightarrow \mathrm{NO}+\mathrm{H})$ and destruction $(\mathrm{NO}+\mathrm{N} \rightarrow$ $\mathrm{N}_{2}+\mathrm{O}$ ) channels are both neutral-neutral reactions with uncertain activation barriers (Gerin et al. 1992). Second, it is unknown whether the main nitrogen reservoir in interstellar clouds is $\mathrm{N}$ or $\mathrm{N}_{2}$, partly because this is difficult to establish since $\mathrm{N}_{2}$ has no rotational line spectrum and $\mathrm{N}$ has no fine-structure lines.

The chemistry of NO is sensitive to temperature, since, in addition to the channels mentioned above, the reaction $\mathrm{NO}+$ $\mathrm{NH} \rightarrow \mathrm{N}_{2} \mathrm{O}+\mathrm{H}$ becomes important at $T \gtrsim 100 \mathrm{~K}$. In the $\mathrm{Sgr} \mathrm{B} 2$ region, $\mathrm{NO}$ is indeed widespread while $\mathrm{N}_{2} \mathrm{O}$ is confined to the warmest parts (Halfen et al. 2001). At temperatures above $\approx 200 \mathrm{~K}, \mathrm{OH}$ is removed from the gas phase by the neutralneutral reaction with $\mathrm{H}_{2}$ into $\mathrm{H}_{2} \mathrm{O}(+\mathrm{H})$. This effect increases the sensitivity of the nitrogen chemistry to temperature because the lack of $\mathrm{OH}$ means that more nitrogen is available in atomic form, leading to an explosion of N-bearing species in hot cores (Rodgers \& Charnley 2001).

Observations of NO in two pre-protostellar cores by Akyilmaz et al. (2007) develop the above picture in more detail. These authors find a depletion of NO toward the centers of these cores and conclude that nitrogen is primarily in atomic form prior to the gravitational collapse of the cores. Based on $\mathrm{N}_{2} \mathrm{H}^{+}$observations, this conclusion was also reached by Maret et al. (2006).

The present paper aims to advance the above discussion by providing the necessary data to obtain accurate estimates of NO abundances from observational data. After an outline of the rotational spectrum of NO (Sect. 2), the calculation of collisional rate coefficients is presented in Sect. 3. The application of these rates in astrophysical radiative transfer calculations is given in Sect. 4, and the main conclusions of this work are in Sect. 5.

\section{Spectroscopy of NO}

Apart from the lowest $(J=1 / 2)$ level, the manifold of rotational levels with quantum number $J$ of the open-shell NO molecule in its ground $X^{2} \Pi$ electronic state is split into two, due to the (electron) spin-orbit coupling. The degree of splitting is governed by the spin-orbit constant $A$. In the case of NO, $A$ is positive, such that the lower manifold, designated by ${ }^{2} \Pi_{1 / 2}$, (Herzberg 1968), corresponds to the states for which the sum, $|\Omega|$, of the projections, $\Lambda$ and $\Sigma$, of, the electronic orbital angular momentum $\boldsymbol{L}$, and the electron spin $S$ on the molecular axis, respectively, is $1 / 2$. The upper manifold corresponds to $|\Omega|=3 / 2$ and is designated by ${ }^{2} \Pi_{3 / 2}$.

In addition, each rotation-spin-orbit level is also doubly degenerate, due to the double degeneracy of a $\Pi$ electronic state. These degenerate pairs have opposite parity, and are usually denoted $e$ and $f$. For a state of doublet multiplicity, the total parity is $+(-1)^{J-1 / 2}$ for the $e$-labeled states and $-(-1)^{J-1 / 2}$ for the $f$-labeled states (Brown 1975). In Hund's case (a), the molecular electronic-rotational states are expanded on a basis of the ${ }^{2} \Pi_{1 / 2}$ and ${ }^{2} \Pi_{3 / 2}$ states. As $J$ increases, the mixing between these states increases, so that $|\Omega|$ no longer remains a good quantum number. In addition, the coupling of the ${ }^{2} \Pi$ ground state with higher-lying $\Sigma$ states lifts the twofold parity degeneracy of these so-called $\Lambda$-doublets.

The nitrogen atom also has a non-zero nuclear spin $(I=1)$. The coupling between $I$ and $J$ results in a splitting of each $\Lambda$ level into 3 hyperfine levels (except for the $J=1 / 2$ level which is split into only 2 levels). Each hyperfine level is designated by a quantum number $F(F=I+J)$ varying between $|I-J|$ and $I+J$.
The theory underlying the rotational spectroscopy of the $X^{2} \Pi$ molecules was discussed by Brown \& Carrington (2003). We used the analysis by these authors of the spectroscopic parameters presented for the NO molecule by Varberg et al. (1999). Good agreement with experiment up to the hyperfine level is achievable, even for a reduced set of only 11 parameters (Brown \& Carrington 2003, p. 535). This should be contrasted with the pioneering study of NO by Meerts \& Dymanus (1972), which required 24 parameters.

In our calculation of the rotational energy levels of NO, we included the centrifugal distortion terms and the nuclear spinrotation term. In order to simplify the calculation, we did not, however, include the $\Delta J= \pm 1$ matrix elements. For the purposes of the present article, the loss of accuracy can be ignored.

The associated matrix elements in the Hund's case (a) parityconserved ${ }^{2} \Pi$ basis can be found in the literature (Gerry et al. 1987; Klisch et al. 1999; Brown \& Carrington 2003 ${ }^{1}$ ).

The energies of the first hyperfine levels are given in Table 1 and shown graphically in Fig. 1. As seen from Table 1, the fine structure levels of the ${ }^{2} \Pi_{3 / 2}$ spin-orbit manifold have high energies $(>180 \mathrm{~K})$ such that the corresponding lines are undetectable in cold interstellar clouds (see Sect. 4 for further discussion). The ordering of single rotational levels and the selection rule for electric dipole transitions, which states that only transitions between levels of opposite parity are allowed, lead to the existence of two series of transitions inside the ${ }^{2} \Pi_{1 / 2}$ spin orbit manifold, the $\Pi^{+}$and $\Pi^{-}$bands, connecting $(e)$ and $(f)$ levels, respectively.

\section{Hyperfine rate coefficients}

Kłos et al. (2008) performed quantum calculations of rate coefficients between fine structure levels of NO in collisions with $\mathrm{He}$ atoms, in which the hyperfine splitting of the NO levels was not included. These calculations used the results of prior ab initio calculations - and the fit thereto - of Kłos and coworkers (Kłos et al. 2000). The calculated potential energy surface (PES) was able to reproduce accurately differential cross sections and the absolute values of steric asymmetries, which were determined experimentally for He-NO by Gijsbertsen et al. (2005) and de Lange et al. (2004), respectively. The degree of agreement suggests that rate coefficients obtained from this PES should be accurate. Scattering calculations were completed using the coupled-states approximation (Alexander 1982), which was shown to be a reasonable approximation to a full closecoupling approach (Alexander 1982). Rate coefficients between the fine structure levels of NO were obtained for temperatures ranging from 1 to $350 \mathrm{~K}$.

Realistic modeling of astrophysical NO line emission requires rate coefficients between hyperfine levels, since modern telescopes usually resolve the corresponding transitions. A full close-coupling (or coupled states) approach is computationally too expensive for molecules with fine and hyperfine structure such as NO. To obtain rate coefficients between hyperfine levels, we adopted an approach that has been used already in astrophysical applications (Guilloteau \& Baudry 1981). It assumes that the hyperfine de-excitation rate coefficients are proportional to the degeneracy $\left(2 F^{\prime}+1\right)$ of the final hyperfine level and completely independent of the initial hyperfine level. This simple method

\footnotetext{
1 More references are cited in Brown \& Carrington (2003). Unfortunately, the list of matrix elements of the nuclear hyperfine terms for ${ }^{2} \Pi$ molecules, pp. 522-524 of this reference, is incomplete and contains several misprints. Corrections are listed at http://physchem. ox . ac. uk/ jmbgroup/.
} 
Table 1. Calculated energies (MHz) for ${ }^{14} \mathrm{~N}^{16} \mathrm{O}$, using the parameters cited in reference (2003) (see also Sect. 2).

\begin{tabular}{|c|c|c|c|c|c|c|c|}
\hline \multicolumn{4}{|c|}{ 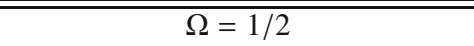 } & $J$ & \multicolumn{3}{|c|}{$\bar{\Omega} \Omega=3 / 2$} \\
\hline 0.5 & 1.5 & 1 & 0.0000 & & & & \\
\hline 0.5 & 0.5 & 1 & 19.9372 & & & & \\
\hline 0.5 & 0.5 & -1 & 225.8576 & & & & \\
\hline 0.5 & 1.5 & -1 & 431.1148 & & & & \\
\hline 1.5 & 2.5 & -1 & 150176.4324 & 1.5 & 0.5 & 1 & 3744741.9932 \\
\hline 1.5 & 1.5 & -1 & 150218.6941 & 1.5 & 0.5 & -1 & 3744743.2251 \\
\hline 1.5 & 0.5 & -1 & 150245.5602 & 1.5 & 1.5 & 1 & 3744788.7339 \\
\hline 1.5 & 0.5 & 1 & 150808.7655 & 1.5 & 1.5 & -1 & 3744789.0772 \\
\hline 1.5 & 1.5 & 1 & 150871.0887 & 1.5 & 2.5 & -1 & 3744862.9825 \\
\hline 1.5 & 2.5 & 1 & 150976.1540 & 1.5 & 2.5 & 1 & 3744865.4416 \\
\hline 2.5 & 3.5 & 1 & 400613.2717 & 2.5 & 1.5 & 1 & 4002610.5845 \\
\hline 2.5 & 2.5 & 1 & 400659.3532 & 2.5 & 1.5 & -1 & 4002610.5910 \\
\hline 2.5 & 1.5 & 1 & 400694.0963 & 2.5 & 2.5 & 1 & 4002641.8444 \\
\hline 2.5 & 1.5 & -1 & 401627.1351 & 2.5 & 2.5 & -1 & 4002643.8739 \\
\hline 2.5 & 2.5 & -1 & 401686.8809 & 2.5 & 3.5 & 1 & 4002685.1382 \\
\hline 2.5 & 3.5 & -1 & 401771.3032 & 2.5 & 3.5 & -1 & 4002691.7806 \\
\hline 3.5 & 4.5 & -1 & 751302.8064 & 3.5 & 2.5 & -1 & 4363566.9430 \\
\hline 3.5 & 3.5 & -1 & 751350.0505 & 3.5 & 2.5 & 1 & 4363570.9933 \\
\hline 3.5 & 2.5 & -1 & 751388.9086 & 3.5 & 3.5 & -1 & 4363590.1554 \\
\hline 3.5 & 2.5 & 1 & 752680.4058 & 3.5 & 3.5 & 1 & 4363597.3591 \\
\hline 3.5 & 3.5 & 1 & 752738.4807 & 3.5 & 4.5 & -1 & 4363619.8299 \\
\hline 3.5 & 4.5 & 1 & 752813.6100 & 3.5 & 4.5 & 1 & 4363633.3427 \\
\hline 4.5 & 5.5 & 1 & 1202242.3389 & 4.5 & 3.5 & 1 & 4827597.3558 \\
\hline 4.5 & 4.5 & 1 & 1202289.8514 & 4.5 & 3.5 & -1 & 4827609.0177 \\
\hline 4.5 & 3.5 & 1 & 1202331.1059 & 4.5 & 4.5 & 1 & 4827615.2970 \\
\hline 4.5 & 3.5 & -1 & 1203974.6460 & 4.5 & 4.5 & -1 & 4827631.2583 \\
\hline 4.5 & 4.5 & -1 & 1204031.4533 & 4.5 & 5.5 & 1 & 4827637.0233 \\
\hline 4.5 & 5.5 & -1 & 1204101.0639 & 4.5 & 5.5 & -1 & 4827660.9693 \\
\hline 5.5 & 6.5 & -1 & 1753429.8449 & 5.5 & 4.5 & -1 & 5394670.8254 \\
\hline 5.5 & 5.5 & -1 & 1753477.2141 & 5.5 & 5.5 & -1 & 5394684.9524 \\
\hline 5.5 & 4.5 & -1 & 1753519.9335 & 5.5 & 4.5 & 1 & 5394694.4674 \\
\hline 5.5 & 4.5 & 1 & 1755509.8632 & 5.5 & 6.5 & -1 & 5394701.3083 \\
\hline 5.5 & 5.5 & 1 & 1755565.6136 & 5.5 & 5.5 & 1 & 5394714.0604 \\
\hline 5.5 & 6.5 & 1 & 1755631.4156 & 5.5 & 6.5 & 1 & 5394740.0785 \\
\hline 6.5 & 7.5 & 1 & 2404862.9493 & 6.5 & 5.5 & 1 & 6064747.2621 \\
\hline 6.5 & 6.5 & 1 & 2404909.9504 & 6.5 & 6.5 & 1 & 6064758.4354 \\
\hline 6.5 & 5.5 & 1 & 2404953.5718 & 6.5 & 7.5 & 1 & 6064770.8082 \\
\hline 6.5 & 5.5 & -1 & 2407284.0641 & 6.5 & 5.5 & -1 & 6064788.0293 \\
\hline 6.5 & 6.5 & -1 & 2407338.8794 & 6.5 & 6.5 & -1 & 6064805.8565 \\
\hline 6.5 & 7.5 & -1 & 2407401.7898 & 6.5 & 7.5 & -1 & 6064829.5764 \\
\hline 7.5 & 8.5 & -1 & 3156538.4631 & 7.5 & 6.5 & -1 & 6837779.0281 \\
\hline 7.5 & 7.5 & -1 & 3156584.9600 & 7.5 & 7.5 & -1 & 6837787.7982 \\
\hline 7.5 & 6.5 & -1 & 3156629.1160 & 7.5 & 8.5 & -1 & 6837797.0202 \\
\hline 7.5 & 6.5 & 1 & 3159293.8907 & 7.5 & 6.5 & 1 & 6837842.8017 \\
\hline 7.5 & 7.5 & 1 & 3159347.8466 & 7.5 & 7.5 & 1 & 6837859.4336 \\
\hline 7.5 & 8.5 & 1 & 3159408.4141 & 7.5 & 8.5 & 1 & 6837881.6950 \\
\hline 8.5 & 9.5 & 1 & 4008452.0515 & 8.5 & 7.5 & 1 & 7713711.5667 \\
\hline 8.5 & 8.5 & 1 & 4008497.9559 & 8.5 & 8.5 & 1 & 7713718.3069 \\
\hline 8.5 & 7.5 & 1 & 4008542.3936 & 8.5 & 9.5 & 1 & 7713724.9171 \\
\hline 8.5 & 7.5 & -1 & 4011534.6339 & 8.5 & 7.5 & -1 & 7713804.9159 \\
\hline 8.5 & 8.5 & -1 & 4011587.7801 & 8.5 & 8.5 & -1 & 7713820.7432 \\
\hline 8.5 & 9.5 & -1 & 4011646.3592 & 8.5 & 9.5 & -1 & 7713842.0904 \\
\hline 9.5 & 10.5 & -1 & 4960597.9352 & 9.5 & 8.5 & -1 & 8692483.8044 \\
\hline 9.5 & 9.5 & -1 & 4960643.1875 & 9.5 & 9.5 & -1 & 8692488.7798 \\
\hline 9.5 & 8.5 & -1 & 4960687.7254 & 9.5 & 10.5 & -1 & 8692493.1479 \\
\hline 9.5 & 8.5 & 1 & 4964000.0686 & 9.5 & 8.5 & 1 & 8692613.9329 \\
\hline 9.5 & 9.5 & 1 & 4964052.4394 & 9.5 & 9.5 & 1 & 8692629.2349 \\
\hline 9.5 & 10.5 & 1 & 4964109.2730 & 9.5 & 10.5 & 1 & 8692650.0405 \\
\hline
\end{tabular}




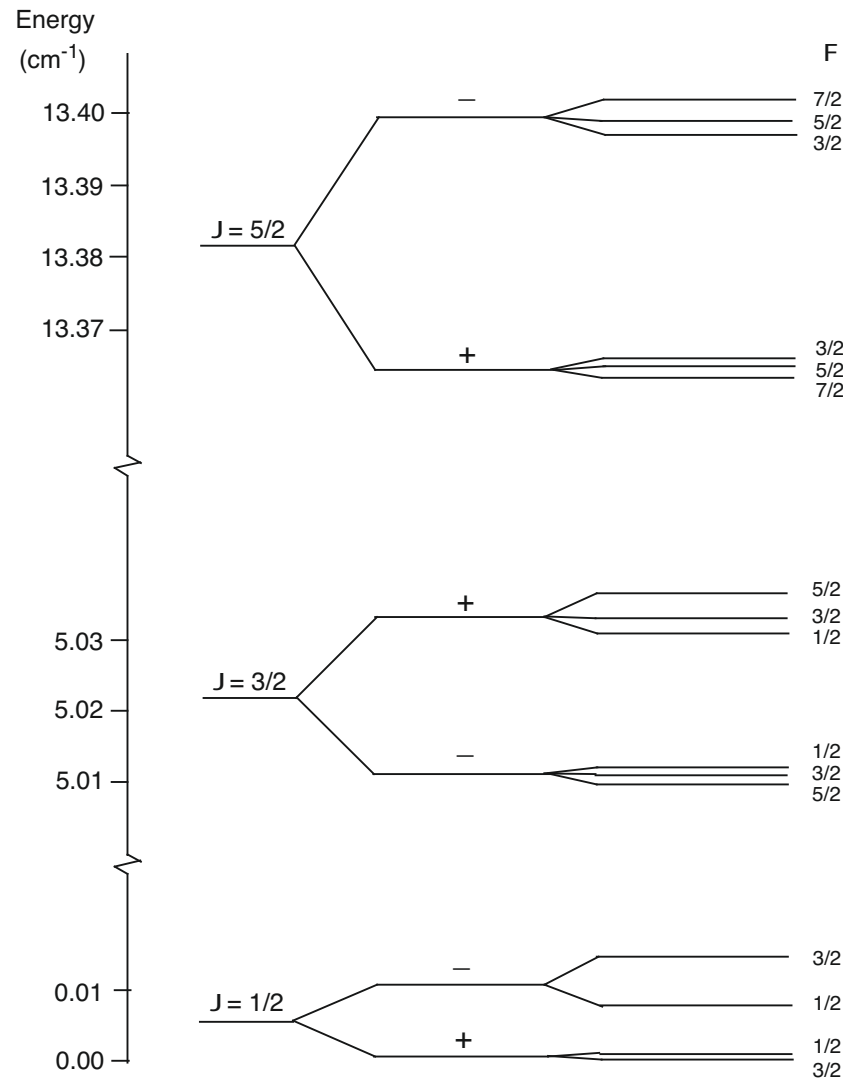

Fig. 1. Hyperfine levels of NO and corresponding rotational energy levels, for $J=1 / 2$ to $5 / 2$ of the ${ }^{2} \Pi_{1 / 2}$ manifold. The + and - signs denote the parity of the lambda-doublet levels. The relationship between the $e$ and $f$ labels and the parity is given in the text.

corresponds to a statistical reorientation of the rotational quantum number $J$ after collision (Alexander \& Dagdigian 1985). Within this approximation, the de-excitation rate coefficients between hyperfine structure levels $\left(k_{J F \rightarrow J^{\prime} F^{\prime}}\right)$ are related to rate coefficients between fine structure levels $\left(k_{J \rightarrow J^{\prime}}\right)$ as follows:

$k_{J F \rightarrow J^{\prime} F^{\prime}}=\frac{\left(2 F^{\prime}+1\right)}{(2 I+1)\left(2 J^{\prime}+1\right)} k_{J \rightarrow J^{\prime}}$

where $I$ is the nuclear spin of the nitrogen atom.

With Eq. (1), we used the NO fine structure rate coefficients published by Kłos et al. (2008) to determine rate coefficients for transitions between NO hyperfine levels. The transitions involve all levels with $J, J^{\prime} \leq 25.5$ and the rates are for temperatures ranging from 10 to $350 \mathrm{~K}$.

Figure 2 presents the temperature variation in $\mathrm{NO}-\mathrm{He}$ rate coefficients for several transitions frequently observed in radio astronomy within the ${ }^{2} \Pi_{1 / 2}$ spin-orbit manifold. The complete set of (de)excitation rate coefficients with $J, J^{\prime} \leq 25.5$ is available online at the LAMDA ${ }^{2}$ website. Excitation rate coefficients can be easily obtained by detailed balance.

As one would anticipate, the rate coefficients display the same propensity rules as observed in calculations without hyperfine structure. The rate coefficients for spin-orbit changing transitions $\left({ }^{2} \Pi_{1 / 2} \leftrightarrow{ }^{2} \Pi_{3 / 2}\right)$ are two orders of magnitude lower than those for transitions within either the ${ }^{2} \Pi_{1 / 2}$ or the ${ }^{2} \Pi_{3 / 2}$ manifold.

\footnotetext{
${ }^{2}$ http://www.strw.leidenuniv.nl/ moldata
}

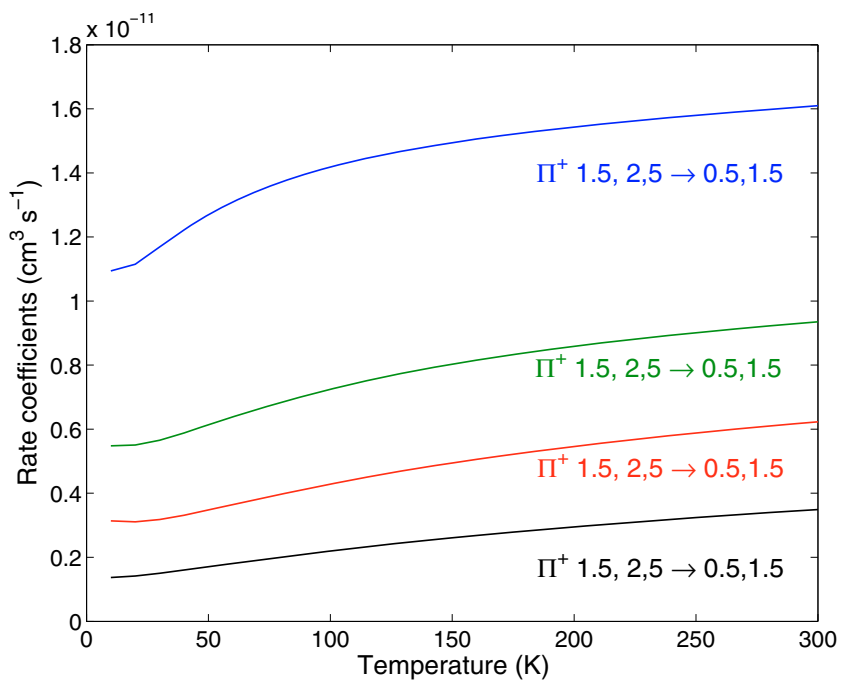

Fig. 2. Temperature variation of the NO-He collisional rate coefficients $\left(k_{J F \rightarrow J^{\prime} F^{\prime}}\right)$. The transitions correspond to spin-orbit conserving transitions within the $\left({ }^{2} \Pi_{1 / 2}\right)$ spin-orbit ladder of NO.

It is important to specify that the rate coefficients presented here do not show the usual hyperfine propensity rules $\Delta J=\Delta F$, since we made use of the approximation represented by Eq. (1) that the rate coefficients are proportional to the degeneracy $\left(2 F^{\prime}+1\right)$ of the final hyperfine level and independent of the initial hyperfine level. Daniel et al. (2005) have demonstrated that this approximation may be indeed inaccurate. Notwithstanding, our rate coefficients are the first to be published for the NO molecule and are probably of the correct order of magnitude, since the accuracy of the underlying NO rate coefficients for transitions between fine structure levels was high.

Finally, the significant abundance of $\mathrm{H}_{2}$ in the interstellar medium implies that this molecule is the primary collision partner for any other species. It is generally assumed (Schöier et al. 2005 ) that rate coefficients with He provide a good estimate of rate coefficients for collision with para- $\mathrm{H}_{2}(j=0)$. For the rotational excitation of SiS, Lique et al. (2008) found that rate coefficients for collisions with para- $\mathrm{H}_{2}(j=0)$ can be up to a factor of 3 higher or lower than those for collisions with He, depending on the selected transition, but that the He rate coefficients scaled by a factor 1.4 provide the correct order of magnitude of the $\mathrm{H}_{2}(j=0)$ rate coefficients. Therefore, the present results provide a reasonable first estimate of collisional rate coefficients for collisions of $\mathrm{NO}$ with $\mathrm{H}_{2}$. On the other hand, it may be unadvisable to use the present He rate coefficients as an estimate of the ortho- $\mathrm{H}_{2}$ rate coefficients, since the $\mathrm{He}$ and ortho- $\mathrm{H}_{2}$ rate coefficients usually differ significantly. Specific calculations with ortho- $\mathrm{H}_{2}$ must be performed.

\section{Astrophysical applications}

\subsection{Model setup}

The RADEX program (Van der Tak et al. 2007) ${ }^{3}$ calculates the intensities of atomic and molecular lines from a uniform medium. Both collisional and radiative processes are taken into account, while optical depth effects are modeled with an escape probability method.

\footnotetext{
${ }^{3}$ http://www.sron.rug.nl/ vdtak/radex/
} 

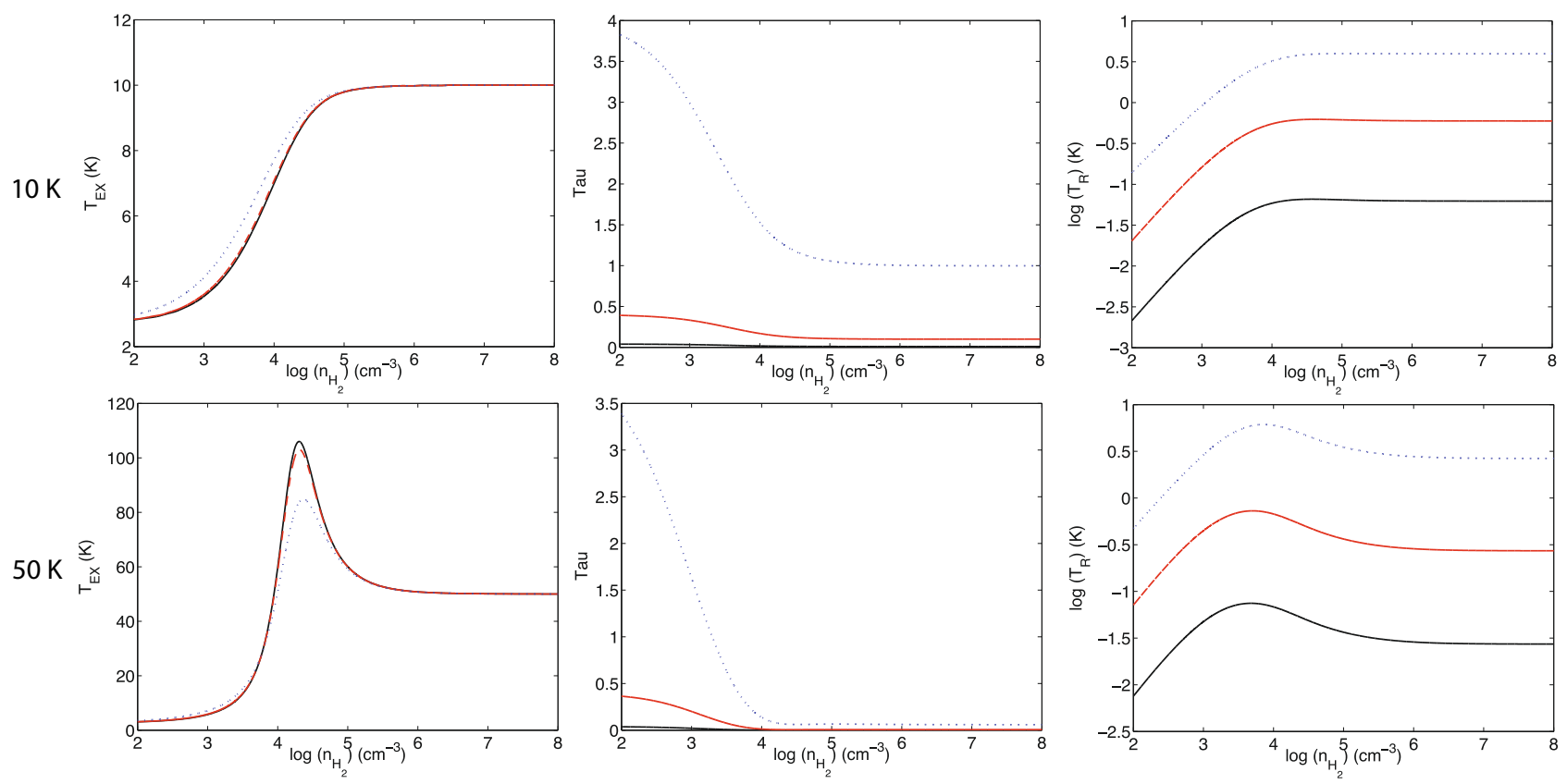

Fig. 3. Excitation temperature (left), optical depth (middle), and emergent intensity (right) for the $150.1764 \mathrm{GHz}$ line of NO, as a function of volume density and column density, for $T_{\text {kin }}=10 \mathrm{~K}($ top $)$ and $T_{\text {kin }}=50 \mathrm{~K}$ (bottom). The column density of NO is $10^{14} \mathrm{~cm}^{-2}$ (black curves), $10^{15} \mathrm{~cm}^{-2}$ (red dashed curves) and $10^{16} \mathrm{~cm}^{-2}$ (blue dot curves).
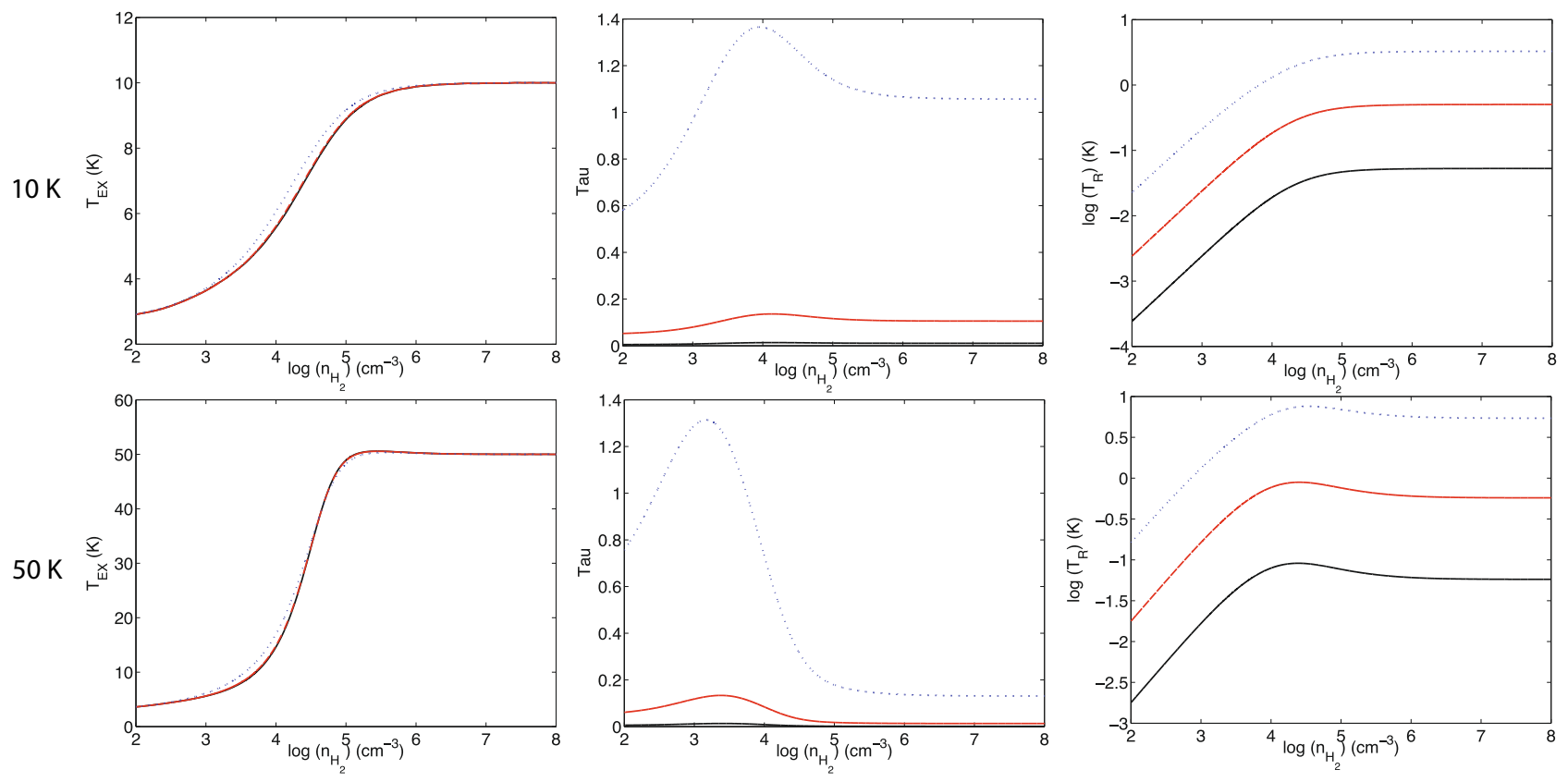

Fig. 4. As previous figure, for the $250.4368 \mathrm{GHz}$ line.

We used RADEX to determine the effect of the new rate coefficients on interpreting observations of interstellar NO. The molecular data-file containing NO energy levels, Einstein A coefficients, and collisional rate coefficients is available from the LAMDA website ${ }^{4}$. The spectroscopic data were taken from the $\mathrm{JPL}^{5}$ database. The spectroscopic data used were not those presented in Sect. 2, but they are in good agreement with the data adopted in Sect. 2 and sufficiently accurate for the purposes of

\footnotetext{
${ }^{4}$ http://www.strw.leidenuniv.nl/ moldata/

5 http://spec.jpl.nasa.gov/ftp/pub/catalog/catform. html
}

our astrophysical modeling. The transitions can be identified by the calculated hyperfine energies in Table 1.

\subsection{Results}

Figures 3 and 4 show some results of our calculations. We focus on the emission in the $50-500 \mathrm{GHz}$ range, which is readily detectable to ground-based telescopes. Furthermore, this discussion only considers the strongest hyperfine component of each $J$-transition, since the trends in the calculations are almost identical for the other, weaker hyperfine components. 
The excitation temperature of the 150 and $250 \mathrm{GHz}$ lines equals the adopted value of the background radiation field $(2.7 \mathrm{~K})$ at low volume densities, and to rise to higher values as collisional excitation becomes more important. At volume densities above a critical value, the excitation temperature approaches the kinetic temperature, at which point the LTE approximation may be used. For the $150 \mathrm{GHz}$ transition, this critical density lies at $\approx 10^{5} \mathrm{~cm}^{-3}$, while for the $250 \mathrm{GHz}$ transition, it is a few times higher. Since many regions of the interstellar medium have densities around or below $10^{5} \mathrm{~cm}^{-3}$, the use of the LTE approximation is not generally justified for NO.

At column densities of below $\sim 1 \times 10^{15} \mathrm{~cm}^{-2}$, the excitation of NO is almost independent of column density. The reason is that due to the low dipole moment of $\mathrm{NO}(0.16 \mathrm{D}$, similar to $\mathrm{CO})$, the ground state lines are optically thin. At high column densities $\left(\gtrsim 10^{16} \mathrm{~cm}^{-2}\right)$, the observed ratios between the various hyperfine components of each $J$-transition may be used to determine their optical depth.

Modern radio telescopes are able to detect an intensity (expressed as an equivalent antenna temperature) of $0.1 \mathrm{~K}$ within minutes, and an intensity of $0.01 \mathrm{~K}$ within hours. Weaker signals may be detected by using wide bandwidths, which is not an option for line radiation, or by using long integration times, which is limited by instrument stability. The figures indicate that current sensitivity limits enable practical measurement of column densities of $\approx 10^{13} \mathrm{~cm}^{-2}$, which implies that NO is observable in a diverse range of astrophysical objects.

The excitation of NO does not vary significantly with temperature: the collisional and radiative (de)excitation rates have only low (although non-negligible) and continuous temperature dependencies. The only exception is the ground state transition, for which the variation in excitation temperature with volume density at $T=50 \mathrm{~K}$ reaches a peak at $T_{\text {ex }}$ at densities immediately below the critical value at which LTE holds. This suprathermal excitation of the ground-state lines is a common effect among linear molecules and was first described by Köppen \& Kegel (1980) for the case of CO. The effect occurs at densities just below the critical value and is caused by common propensity rules for the collision rates. The $\Delta J=2$ rates tend to be higher than those for $\Delta J=1$, which leads to an inversion of the populations of the lowest energy levels of these molecules. The main observable consequence is an increased uncertainty in column densities derived from ground-state lines at densities just below critical. In this regime, the excitation temperature is extremely sensitive to the volume density.

As for the other NO lines in the $50-500 \mathrm{GHz}$ range, we find that all lines in the $\Omega=1 / 2$ ladder are detectable $\left(T_{R}>10 \mathrm{mK}\right)$ for column densities $>10^{13} \mathrm{~cm}^{-2}$ and any temperature. In contrast, the lines in the higher-lying $\Omega=3 / 2$ ladder require higher column densities $\left(>10^{14} \mathrm{~cm}^{-2}\right)$ and temperatures $(>50 \mathrm{~K})$ to be detectable within reasonable integration times.

Figure 5 shows another means of using observations of NO line emission: the intensity ratio of the 150 and $250 \mathrm{GHz}$ lines may be used to constrain the kinetic temperature and/or the density of the emitting region. The figure shows a behavior which is characteristic of linear molecules (cf. Appendix C of Van der Tak et al. 2007). At low densities (below the critical value of both lines), the intensity ratio is a measure of the density: the stronger the lower-excitation line, the lower the density. At high densities, the intensity ratio becomes a measure of temperature: the stronger the lower-excitation line, the lower the temperature. If measurements of three or more lines (with different critical densities) are available, both temperature and density can be constrained (see Van der Tak et al. 2007, for examples).

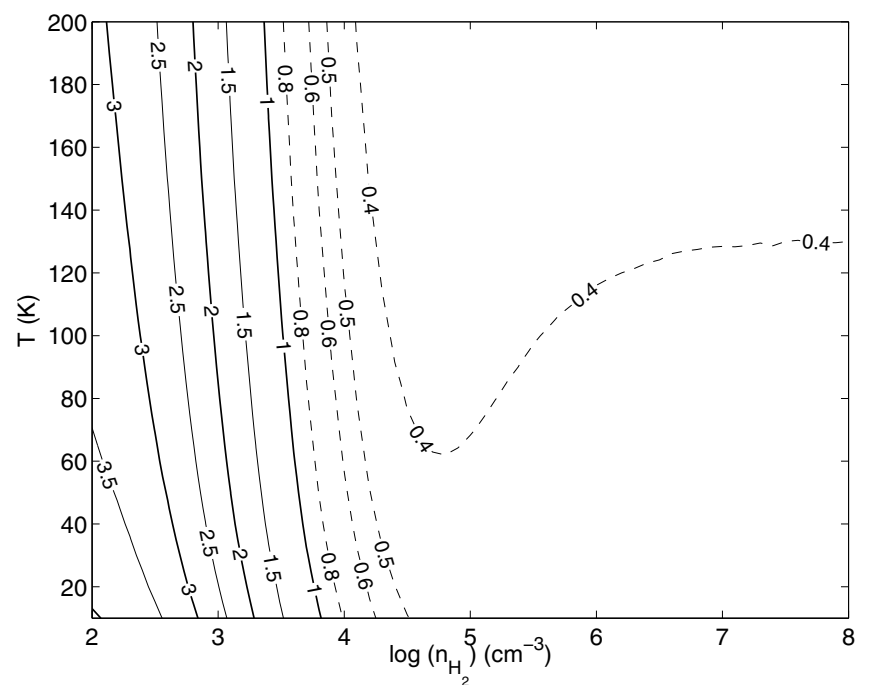

Fig. 5. Calculated intensity ratio of the 150 and $250 \mathrm{GHz}$ lines of NO as a function of kinetic temperature and volume density. The column density of $\mathrm{NO}$ is $10^{14} \mathrm{~cm}^{-2}$.

\section{Conclusions}

Observed column densities of NO are measured typically to be in the range $10^{15}-10^{16} \mathrm{~cm}^{-2}$ in diverse regions as the pre-stellar cores L 183 (L 134N) and L 1544, the Orion region of high-mass star formation, the Sgr B2 cloud at the center of our Galaxy, and the nucleus of the starburst galaxy NGC 253. Assuming LTE in these environments, preliminary estimates of the NO abundance are about $10^{-8}$ with only small variation (Akyilmaz et al. 2007; Ziurys et al. 1991; Halfen et al. 2001; Martín et al. 2006). However, at these column densities, the ground-state lines of NO (which are those most frequently observed) will be at least moderately optically thick. At least part of the gas in these regions is at densities of below $10^{5} \mathrm{~cm}^{-3}$, which is the regime in which radiation competes with collisions in the excitation of NO. Our calculations therefore demonstrate the need for a full non-LTE treatment when interpreting observations of interstellar NO.

The molecular data presented here enable accurate determinations of NO abundances for a wide range of temperatures, densities, and column densities. One question of interest is a possible enhancement of NO in shocked gas, which may be expected given the temperature dependence of the main production routes of NO, which are neutral-neutral reactions with likely activation barriers. However, it is also possible that the oxygen in shocks is converted entirely to $\mathrm{OH}$ and $\mathrm{H}_{2} \mathrm{O}$. Observations of $\mathrm{NO}$ will help to answer this question, since they have the advantage being able to be performed from the ground, unlike those of $\mathrm{OH}$ or $\mathrm{H}_{2} \mathrm{O}$.

Observations of highly excited NO lines with the HIFI instrument onboard ESA's Herschel Space Observatory, scheduled for launch in 2009, will address the possible enhancement of NO in hot gas. A few years later, high-resolution observations of NO with the Atacama Large Millimeter Array (ALMA) will enable detailed investigations of nitrogen and oxygen chemistry in a wide range of astrophysical environments.

Acknowledgements. F.L., J.K. and M.H.A. would like to acknowledge financial support from the US National Science Foundation under grant CHE0413743. F.v.d.T. acknowledges support from the Netherlands Research School for Astronomy (NOVA). 


\section{References}

Akyilmaz, M., Flower, D. R., Hily-Blant, P., Pineau Des Forêts, G., \& Walmsley, C. M. 2007, A\&A, 462, 221

Alexander, M. H. 1982, J. Chem. Phys., 76, 5974

Alexander, M. H., \& Dagdigian, P. J. 1985, J. Chem. Phys., 83, 2191

Brown, J. M., \& Carrington, A. 2003, Rotational Spectroscopy of Diatomic Molecules (Cambridge)

Brown, J. M., Hougen, J. T., Huber, K.-P., et al. 1975, J. Mol. Spectrosc., 55, 500

Castor, J. I. 1970, MNRAS, 149, 111

Daniel, F., Dubernet, M.-L., Meuwly, M., Cernicharo, J., \& Pagani, L. 2005, MNRAS, 363, 1083

De Lange, M. J. L., Stolte, S., Taatjes, C. A., et al. 2004, J. Chem. Phys., 121 11691

Gerin, M., Viala, Y., Pauzat, F., \& Ellinger, Y. 1992, A\&A, 266, 463

Gerry, M. C. L., Merer, A. J., Sassenberg, U., \& Steimle, T. C. 1997, J. Chem. Phys., 86, 4754

Gijsbertsen, A., Linnartz, H., Rus, G., et al. 2005, J. Chem. Phys., 123, 224305

Guilloteau, S., \& Baudry, A. 1981, A\&A, 97, 213

Halfen, D. T., Apponi, A. J., \& Ziurys, L. M. 2001, ApJ, 561, 244

Herzberg, G. 1968, Spectra of Diatomic Molecules (Van Nostrand: Princeton)

Klisch, E., Belov, S. P., Schieder, R., Winnewisser, G., \& Herbst, E. 1999, Mol. Phys., 97, 65
Kłos, J., \& Lique, F. 2008, MNRAS, 390, 239

Kłos, J., Chałasiński, G., Bukowski, R., Cybulski, S. M., \& Berry, M. T. 2000, J. Chem. Phys., 112, 2195

Kłos, J., Lique, F., \& Alexander, M. H. 2008, Chem. Phys. Lett., 455, 1

Köppen, J., \& Kegel, W. H. 1980, A\&AS, 42, 59

Lique, F., \& Kłos, J. 2008, J. Chem. Phys., 128, 034306

Lique, F., Toboła, R., Kłos, J., et al. 2008, A\&A, 478, 567

Maret, S., Bergin, E. A., \& Lada, C. J. 2006, Nature, 442, 425

Martín, S., Mauersberger, R., Martín-Pintado, J., Henkel, C., \& García-Burillo, S. 2006, ApJS, 164, 450

Meerts, W. L., \& Dymanus, A. 1972, J. Mol. Spectrosc., 44, 320

Rodgers, Charnley 2001, ApJ, 546, 342

Schöier, F. L., van der Tak, F. F. S., van Dishoeck, E. F., \& Black, J. H. 2005, A\&A, 432, 369

Sobolev, V. V. 1958, Theorical Astrophysics, ed. V. A. Ambartsumyan (London: Pergamon Press Ltd.), Chap. 29

Sobolev, V. V. 1960, Moving envelopes of stars (Harvard University Press)

Van der Tak, F. F. S. 2005, Proc. IAU Symp., 227

Van der Tak, F. F. S., Black, J. H., Schier, F. L., Jansen, D. J., \& van Dishoeck, E. F. 2007, A\&A, 468, 627

Varberg, T. D., Stroh, F., \& Evenson, K. M. 1999, J. Mol. Spectrosc., 196, 5

Ziurys, L. M., McGonagle, D., Minh, Y., \& Irvine, W. M. 1991, ApJ, 373, 535 